\title{
Research of thickness by using subminiature eddy-current transducers
}

\author{
Sergey F. Dmitriev, Vladimir N. Malikov*, and Anatoly M. Sagalakov \\ Altai State University, Faculty physics and technology, 65604 Barnaul, Russia
}

\begin{abstract}
On the basis of an eddy-current transducer of a transformer type, a measuring system is made, which makes it possible to estimate the possibility of using the eddy current method for measuring the thickness of conductive and dielectric coatings placed on a conductive base. The purpose of this work was to evaluate the possibility of using only an amplitude eddycurrent method for determining the thickness of a conductive or dielectric coating on a conductive base and estimating the error of such measurements. The design of the measuring system and measurement techniques is described. The article presents data demonstrating the dependence of the amplitude part of the signal on objects of different thicknesses; limiting object dimensions at which these measurements are expedient are experimentally established.
\end{abstract}

\section{Introduction}

To control the thickness of electrically conductive sheets, films, plates, coatings on them, walls of pipes, cylindrical and spherical vessels, and determine the air gap between plates of the same material, thickness gauges based on the eddy-current method are used. Conductive and dielectric coatings are widely used in such industries as aerospace, shipbuilding, oil and gas. Such coatings are often used as a protection against thermal and corrosive effects, for electrical insulation [1]. However, in the process of application and operation of such coatings, problems arise with different thicknesses of the applied coating. Another potentially dangerous aspect is corrosion under the coatings themselves in case of their poor-quality application. Therefore, non-destructive testing of coatings applied to metals used in extreme conditions and in equipment operating under extreme conditions is especially urgent.

At present, thermal, microwave, ultrasonic and eddy-current testing methods are used for non-destructive testing of coatings [2, 3]. Eddy-current control has significant advantages over other methods: it is resistant to extreme operating conditions, the measurement is noncontact, and its cost is relatively low [4]. For example, to estimate the conductive coating thickness, Takashi et al [5] established the possibility of estimating the thickness of a coating based on nickel sputtered on stainless austenitic steels (type 304). The limit of the measured coating thickness was $22 \mu \mathrm{m}$.

Barbosa [6] derived a formula that allows obtaining the value of the thickness of galvanized steel, depending on the impedance of the coil of an eddy-current transducer. Yang

\footnotetext{
*Corresponding author: osys11@gmail.com
} 
[8] and Tai $[3,7]$ used pulse control methods to determine conductivity and coating thickness on a metal base plate under magnetization conditions.

In the eddy-current thickness studies of an object, which is a dielectric or conductive top layer (coating) located on a conducting base (base plate), control based on phase $\varphi$ measurements of the signal introduced into the transducer is usually used [9]. The thickness of the top layer $d$ is determined by the phase changes during scanning. An attachable transducer, as a rule, is a transducer of a transformer type with three windings. The exciting winding creates an excitation field, and the measuring and compensating windings are intended for measurements. The phase is measured relative to the sinusoidal excitation current of the transducer; in [10], in order to increase the accuracy of measurements, the EMF induced in the compensating winding of the transducer was used as the reference signal. The phase of the EMF introduced into the measuring winding depends on the geometric parameters of the transducer, the excitation current frequency $f$, the gap between the transducer and the object of control (OC), the electrical conductivities of the coating $\sigma 1$ and the base $\sigma 2$, and also the relative magnetic permeability of the base.

In practice, variations in the value of $\sigma 1$ are usually observed when moving from point to point along the surface of the OC, in the transition from one OC to another. This leads to variations in the value of $\varphi$ with an unchanged value of the coating thickness $d$, which causes an error in determining $d$. At the same time, the amplitude, phase, amplitude-phase detuning from the influence of the variations of $\sigma 1$ on the results of measurements of $d$ is practically impossible [11]. The influence of variations of $\sigma 1$ on the eddy-current thickness gauge can be reduced by lowering the frequency f of the excitation current of the transducer, but in this case, the interfering factors can be variations in the thickness of the base plate and the magnetic permeability of its material. As a result, with a small thickness of the base plate, it is necessary to choose a relatively high operating frequency $f$ of the thickness gauge and apply the method of detuning from the influence of the variations of $\sigma 1$ on the measurement results of $d$. In this way, the value of $\sigma 1$ is determined on the basis of measuring the amplitude A or (and) phase $\varphi$ of introduced into the attachable EMF transducer at such a relatively high frequency of the excitation field $\mathrm{f}$ at which the electromagnetic parameters of the base plate do not influence the measurement results. After this, the measurements of $\varphi$ or A of the introduced EMF are made at a lower frequency $f$, when the measured parameters contain information on the thickness of the coating $\mathrm{d}$. At the same time, when analysing the measurement results of $\varphi$ or A in order to determine the value of $\mathrm{d}$, the found value $\sigma 1$ is taken into account. An example of the implementation of this method of control is given in [12].

The purpose of this work was to evaluate the possibility of using only an amplitude eddycurrent method for determining the thickness of a conductive or dielectric coating on a conductive base and estimating the error of such measurements. The possibility of determining the thickness of conductive objects which consist of several alternating conductive and nonconductive layers and monolithic conductive objects was also investigated.

\section{Description of the system}

The subminiature eddy-current transducer [13-16] was developed for local studies of the thickness of various coatings in experiments, as well as to determine the effect of various coatings on the magnitude of the output signal.

The developed subminiature eddy-current transducer is a core with the following windings applied to it: exciting, measuring and compensating. The core and winding structure is impregnated with the compound and is enclosed in a corundum washer, so it is resistant to mechanical influences.

For testing on model objects from various conductive materials, a subminiature eddycurrent transducer was used, which is connected to a personal computer via a sound card. The sound card is used as a generator and converter of a signal sent to the exciting winding. 
The software allows one to control the value of the signal applied to the exciting winding, and also allows reading the voltage values from the measuring winding, which, taking into account the calibration, are converted into conductivity values. The developed software allows measuring the thickness of conductive and dielectric non-ferromagnetic coatings and conductive materials

To wind the turns, copper wire 5 microns thick is used. The core is made of ferrite 2000 $\mathrm{NMZ}$ with an initial magnetic permeability value of 500 and has a pyramidal shape. The characteristics of the developed transducer make it possible to achieve high localization of control, namely, to localize the field within $2500 \mu \mathrm{m} 2$. This device provides a significant depth of penetration of the field into the object under investigation up to the values of $5 \mathrm{~mm}$ (at frequencies of about $500 \mathrm{~Hz}$ ).

Special software written in the $\mathrm{C}++$ language for the Windows operating system allows controlling the signal on the exciting coil and receiving the signal from the measuring winding. By means of a special mixer, voltage is applied to the exciting winding of the transducer and the level and frequency of the sinusoidal signal of the generator are set.

The use of a sound card allows broadening the frequency limits of the signals fed to the exciter winding significantly, which in turn allows significantly expanding the measurement range.

\section{Results and discussion}

In order to test the developed measuring system, the aluminium coating having a different thickness and placed on a copper base $3 \mathrm{~mm}$ thick was scanned. Figure 1 shows the dependence of the signal value on the thickness of the aluminium coating on a nonferromagnetic basis. As the thickness of the non-ferromagnetic coating is increased to $1200 \mu \mathrm{m}$, the signal value decreases from 28 to $22 \mathrm{mv}$. In the range from 750 to $1500 \mu \mathrm{m}$, the signal is much smaller than the signal from the monolith, which can be explained by the insufficient thickness of the coating. However, for coating thicknesses from 1500 to $2500 \mu \mathrm{m}$, the magnitude of the signal is constant and corresponds to the magnitude of the field from the monolith.

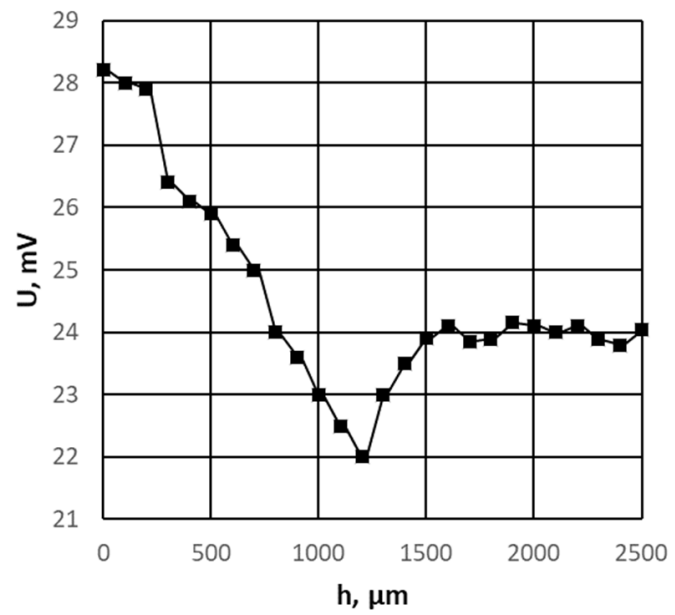

Fig. 1. Dependence of the signal value on the thickness of the aluminium coating on a copper base.

The following experiment shows the dependences illustrating the change in the signal from the thickness of the layered coating with the alternation of layers of foil and polyethylene placed on a copper base. The object of the study was the alternation of layers of aluminium foil $20 \mu \mathrm{m}$ and polyethylene $20 \mu \mathrm{m}$. 
Figure 2 presents the dependence of the signal intensity on the thickness of the layered coating with the alternation of layers of foil and polyethylene placed on a copper base. At thicknesses from 0 to $100 \mu \mathrm{m}$, the magnitude of the signal from the base varies from 29 to 24 $\mathrm{mv}$, and at thicknesses from 100 to $250 \mu \mathrm{m}$ the character of the signal change is gentler. In the range from $250 \mu \mathrm{m}$ to $400 \mu \mathrm{m}$ the signal varies from 26 to $23 \mathrm{mv}$, which is due to the contribution of the signal from the layered coating and to the reduction of the contribution of the signal from the copper base plate.

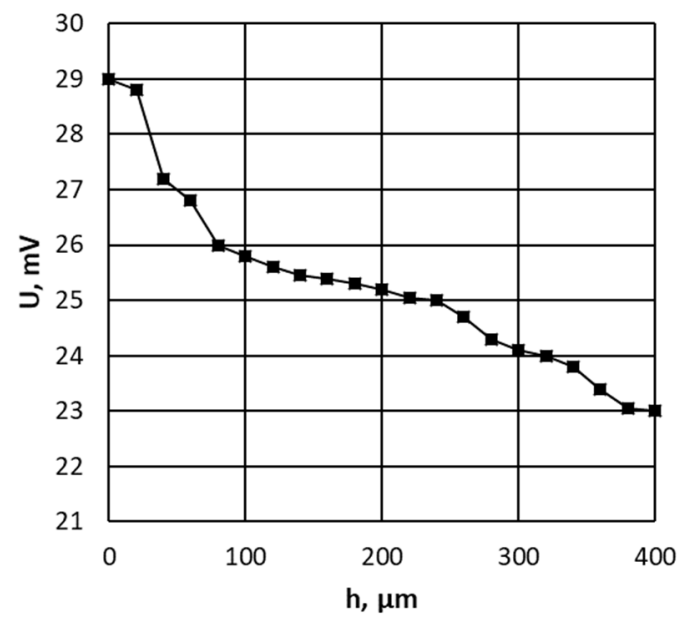

Fig. 2. Dependence of the signal intensity during the scanning of a layered structure.

In the third test experiment, a sample was taken representing a single object of aluminium of different thicknesses. Figure 3 shows the dependence of the value of the signal on the thickness of the sample of aluminium. With the increasing sample thickness, the contribution of the deeper layers of the sample to the signal amplitude increases. When the thickness varies from 100 to $1200 \mu \mathrm{m}$, the signal value increases from 7 to $25 \mathrm{mv}$. When the thickness varies from 1200 to 2200 microns, the magnitude of the signal is constant and corresponds to the value of the amplitude from the monolith $(25.5 \mathrm{mv})$.

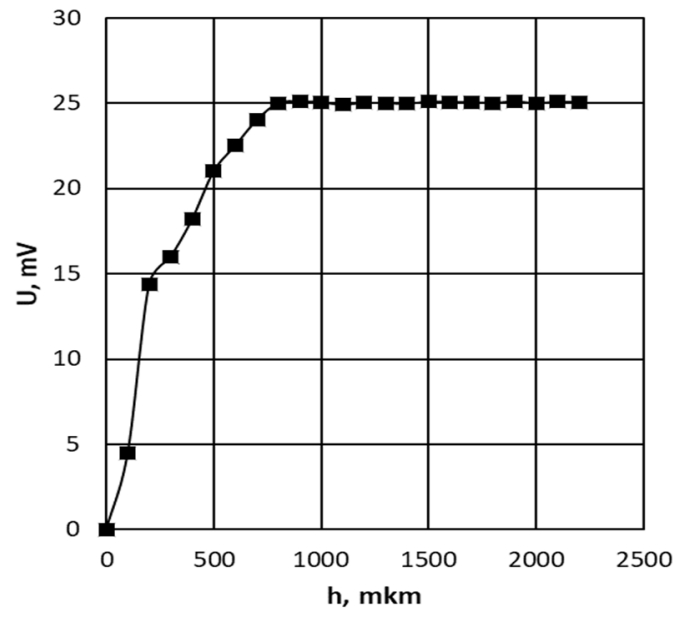

Fig. 3. Dependence of the signal on the thickness of the aluminium sample. 
In the final study, the control object was a copper plate and a dielectric coating. As a dielectric coating, a layer of varnish was used. The copper sample was made of a solid piece of copper. A layer of dielectric coating was applied to the controlled object.

A series of experiments was also conducted with copper samples and a paint and varnish coating. On the pre-cut samples of copper, a layer of paint and varnish coating was applied. As can be seen from the dependence of the signal amplitude on the thickness of the dielectric coating (Figure 4), the magnitude of the output signal decreases rapidly with increasing coating thickness. This dependence can be approximated by an exponential function:

$$
y=A_{1} e^{-\frac{x}{t}+y_{0}}
$$

The graph of this dependence and the parameters of the approximating function are shown in Figure 4. As can be seen from the Figure, the signal decreases exponentially with increasing thickness of the dielectric coating.

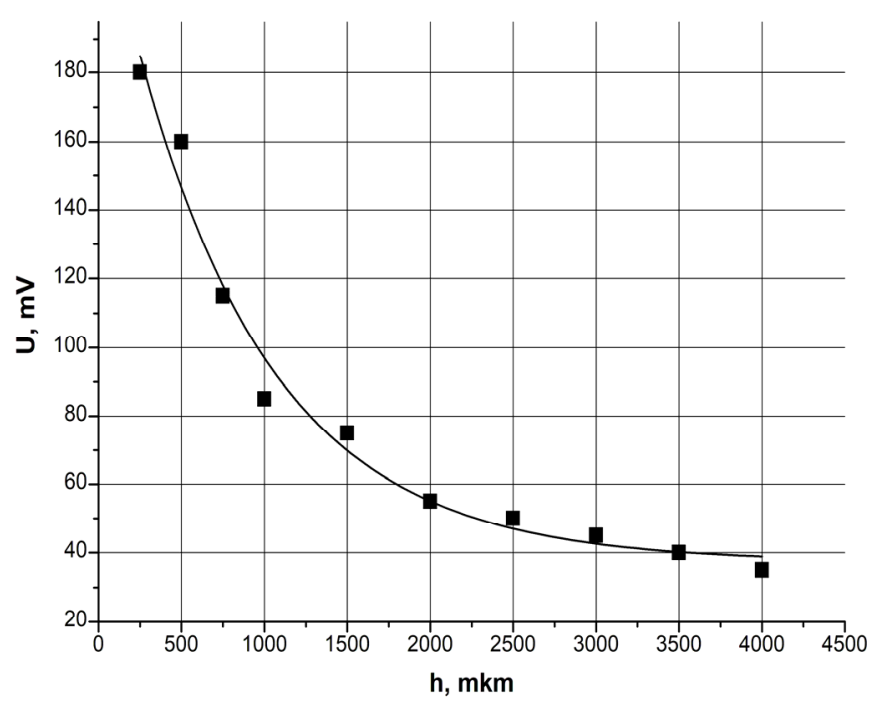

Fig. 4. Dependence of the eddy-current transducer response on the thickness of the dielectric coating.

\section{Conclusions}

The developed measuring system was used to study objects representing conductive and nonconductive coatings placed on a conductive base, and also to measure the thickness of the monolithic conductive objects. It is established that the thickness of the coating affects the signal of the eddy-current transducer. This allows using a perspective amplitude control method of such objects for accurate measurements of the thickness of conductive and nonconductive coatings, as well as other objects.

In conclusion, we note that these test experiments were conducted to determine the effect of various objects on the output signal of an eddy-current transducer. The distortions of the eddy-current transducer signals during scanning of coatings are determined and it is established that these distortions depend on the type of coatings and the plate base.

The reported study was funded by RFBR according to the research project № 18-38-00272. 


\section{References}

1. A. Ptchelintsev, B. Halleux, Rev. Sci. Instrum., 69(3), 1488 (1998)

2. M. T. Ghasr, M. J. Horst, M. Lechuga, IEEE Trans. Instrum. Meas., 64(10), 2802 (2015)

3. C. C. Tai, J. H. Rose, M. Lechuga, Rev. Sci. Instrum., 67(11), 3965 (1996)

4. B. Halleux, S. Limburg, Rev. Sci. Instrum., 68(9), 3533 (1997)

5. Y. Takahashi, Int. J. Appl Electromagn. Mech., 39(1), 419 (2015)

6. C. F. Barbosa, IEEE Trans. Magn., 50(7), 1 (2014)

7. C. C. Tai, Rev. Sci. Instrum., 71(8), 3161 (2000)

8. H. C. Yang, C. C. Tai, Meas. Sci. Technol., 13(8), 1259 (2002)

9. P. Neumeier, V Mire Nerazrushayushchego Kontrol., 2(40), 29 (2008)

10. V. A. Sjas'ko, A. S. Bulatov, M. U. Koroteev, Eddy Current Meter, Patent RF 2384839 (2011)

11. A.S. Bakunov, V.A. Kaloshin, Contr. diagn., 211, 27 (2016)

12. N.G. Bogdanov, V.A. Prihod'ko, A. I. Suzdal'cev, Eddy Current Method of Dual Frequency Control of Products, Patent RF 2184931 (2002)

13. S. F. Dmitriev, V. N. Malikov, A. M. Sagalakov, MATEC Web of Conf., 106, 1 (2017)

14. S. F. Dmitriev, A. V. Ishkov, V. N. Malikov, A. M. Sagalakov, Mat. Sci. For., 906, 147 (2017)

15. S. F. Dmitriev, V. N. Malikov, A. M. Sagalakov, L. I. Shevtsova, Welding Int., 31(8), 608 (2017)

16. S. F. Dmitriev, V. N. Malikov, A. M. Sagalakov, M. A. Davydchenko, AIP Conf. Proc., 1785, 1 (2016) 